\section{INSTRUMENTED LUMBAR SPINE SURGERY UNDER SPINAL ANESTHESIA AND ERECTOR SPINAE BLOCK IN A STAGE IV LUNG CANCER PATIENT - A CASE-REPORT}

AP Martins Pereira*, FJ Moutinho Teixeira, JC Patrício Sampaio, M de Sá Rodrigues Moura Vieira. Centro Hospitalar de Trás-os-Montes e Alto Douro, Vila Real, Portugal

\subsection{6/rapm-2021-ESRA.93}

Background and Aims Severe pulmonary conditions in patients undergoing surgery place them at greater risk of adverse outcomes and alternatives to general anaesthesia may be encouraged. Prone spinal surgery is customarily performed under GA. We describe the use of regional anaesthesia techniques to successfully circumvent post-operative pulmonary events in a patient with advanced disseminated lung cancer.

Methods A 63-year-old man, ASA IV, with debilitating stage IV lung cancer with pleural and mediastinic involvement, pulmonary artery invasion and adrenal, cerebellum and lumbar vertebrae metastatic disease, presents with severe disabling pain, in need for spinal surgery due to vertebral pathological fracture. After obtaining patient's consent, an ultrasoundguided bilateral erector spinae block at L2 level (with ropivacaine and dexamethasone) and a spinal blockade (with hyperbaric bupivacaine and sufentanil) were performed. IV infusions of low-dose propofol, ketamine and lidocaine were maintained during surgery.

Results Patient underwent uneventful L2 vertebroplasty and L1-L3 spinal fusion instrumentation. Quality and extension of the block were adequate and allowed surgery. Patient was comfortable during and after the procedure and safely discharged from anesthesia care. Injury-related pain got manageable, and patient was satisfied with the approach. No postoperative complications were observed.

Conclusions In addition to several other reasons like decreased blood loss, less postoperative nausea and vomiting and better pain control comparing with $\mathrm{GA}^{1}$, regional anaesthetic strategies appear to be useful alternatives for spinal surgery including instrumented procedures - in patients with important pulmonary comorbidities, namely late stage lung cancer, who are at substantial risk for postoperative complications, avoiding further respiratory compromise in these frail patients.

\section{ULTRASOUND-GUIDED BOTOX INJECTIONS FOR ABDOMINAL MUSCULATURE RELAXATION TO FACILITATE LARGE VENTRAL HERNIA CLOSURE}

M Billstrand* , C Arndt. University of New Mexico, Albuquerque, USA

\subsection{6/rapm-2021-ESRA.94}

Background and Aims We present a case of regional anesthesiologists using ultrasound-guidance to strategically place Botox injections into the abdominal musculature to facilitate closure of a large ventral hernia. A male patient with complex abdominal surgical history that had previously failed abdominal wall reconstruction was scheduled for a reattempt at ventral wall closure and the surgeon consulted anesthesiology for Botox injections.

Methods Three weeks before the surgery, the patient presented to anesthesiology. His abdominal girth was measured at the umbilicus while relaxing $(88.5 \mathrm{~cm})$ and while contracting $(97 \mathrm{~cm})$ his musculature. Measurement of his defect across the umbilicus by palpation was $16 \mathrm{~cm}$. After monitors, IV placement and sedation the patient received Botox injections. Under

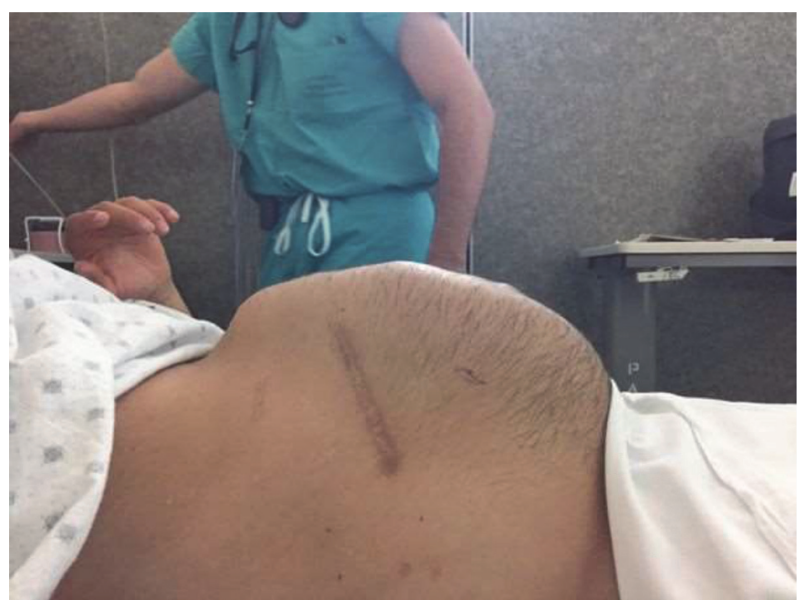

Abstract 94 Figure 1

ultrasound-guidance we identified three muscle layers; external oblique, internal oblique and transverse abdominis. We choose three sites per side for injection; a superior, middle and inferior site. Using a $22 \mathrm{~g}$ Pajunk needle we placed $8 \mathrm{ml}$ of Botox $2 \mathrm{U} / \mathrm{ml}$ per muscle layer for a total of 300 units of Botox. The patient tolerated the procedure well.

Results At the time of surgery, the patient's abdominal girth at the umbilicus with relaxation was $(91 \mathrm{~cm})$ and contraction $(105 \mathrm{~cm})$. Palpation of the hernia was measured at the umbilicus at $19 \mathrm{~cm}$. Demonstrating relaxation of abdominal musculature. The patient proceeded to surgery with successful closure. Conclusions As the technological advances in ultrasound have advanced so has ultrasound-guided regional anesthesia and the role of the regional anesthesiologist. We bring forward this case report as an example of regional anesthesiologists' expertise being utilized outside the traditional setting to facilitate better outcomes for surgical patients.

\section{MOVING THE NEEDLE IN U.K. ANAESTHESIA TRAINING: THE INITIATIVE TO PROMOTE UNIVERSAL COMPETENCE IN REGIONAL ANAESTHESIA}

L Suntharanathan*, A Loughnan, D Tong, A Devlin. King's College Hospital, London, UK

\subsection{6/rapm-2021-ESRA.95}

Background and Aims Historically regional anaesthesia has been an optional unit of UK higher anaesthesia training. However, 2019 U.K. day surgery guidelines recommend all anaesthetists should be familiar with regional techniques ${ }^{1}$ and a recent Anaesthesia editorial proposed a list of 'Plan A' regional blocks, that all anaesthetists should be competent in ${ }^{2}$.

These publications demonstrate the necessity for regional anaesthesia to develop from a subspecialist skill to an essential one. To reflect this, the incoming 2021 UK anaesthesia curriculum requires trainees to independently perform ultrasoundguided regional anaesthesia to receive a certificate of completion of training ${ }^{3}$.

We aimed to investigate the current level of training in ultrasound-guided regional techniques amongst higher trainees in our institution in line with the expectations of these publications.

Methods A survey of regional anaesthesia training and ability to perform ultrasound-guided regional techniques was 
performed amongst higher trainees at our tertiary-centre teaching hospital.

Results $100 \%$ response rate was obtained from 23 trainees. $43 \%$ and $60 \%$ felt confident in performing a brachial plexus block and lower limb block, respectively. 30\% felt confident performing regional anaesthesia for awake surgery whilst $17 \%$ reported sufficient opportunities in general training to develop competence in both upper and lower limb regional techniques. Conclusions Despite applications of regional anaesthesia becoming more prevalent, it currently remains a subspecialist skill. The mandate for regional anaesthesia proficiency in the new UK curriculum aims to address this disparity and improve equity of patient care. Our findings suggest there will need to be significant promotion of regional anaesthesia within training to enable universal competence.

\section{FEASIBILITY OF PRE-ANESTHETIC TELEMEDICINE CONSULTATION IN ELECTIVE ORTHOPEDIC SURGERY: EXPERIENCE DURING THE COVID-19 PANDEMIC}

${ }^{1} \mathrm{~F}$ Le Sache*, ${ }^{2} \mathrm{C}$ Naudin, ${ }^{2} \mathrm{M}$ Merzoug, ${ }^{1} \mathrm{M}$ Bucciero, ${ }^{1} \mathrm{D}$ Barouk, ${ }^{1} \mathrm{~S}$ Bloc. ${ }^{1} \mathrm{Clinique}$ Drouot Sport, Paris, France; ${ }^{2}$ Clinical Research Department, Neuilly-sur-Seine, France

\subsection{6/rapm-2021-ESRA.96}

Background and Aims Telemedicine is becoming more and more used for medical consultation. During the post-COVID19 period this mode was supported by the French Society of Anesthesia to reduce the risk of viral transmission $(1,2)$. The aim of this study was to determine the rate of successful telemedicine preoperative anesthesia consultation.

Methods This observational, single-center, prospective study, included 267 patients scheduled for elective orthopedic surgery. They were invited to choose either virtual (TeleMed-C) or face-to-face (Face-Face-C) anesthetic preoperative consultation. Every TeleMed-C was carried out through a dedicated website. The Patient Environment Quality Index (PEQI) during TeleMed-C was assessed. Patient satisfaction and opinion about TeleMed-C were assessed using a 5-point Likert scale. The use of a third-party facilitator was noted.

Results The success of TeleMed-C without cancellation or postponement of surgery was $89.5 \%$. A third-party facilitator was present in $46 \%$ of the patients over 65 yr. TeleMed-C happened in an adapted environment (98\%) which led to a high level of patient satisfaction (97\%) and a very high opinion (93\%).

Conclusions TeleMed-C is a preoperative anesthetic consultation method which could be widely highly selected by the patients, without increasing the risks of postponed or

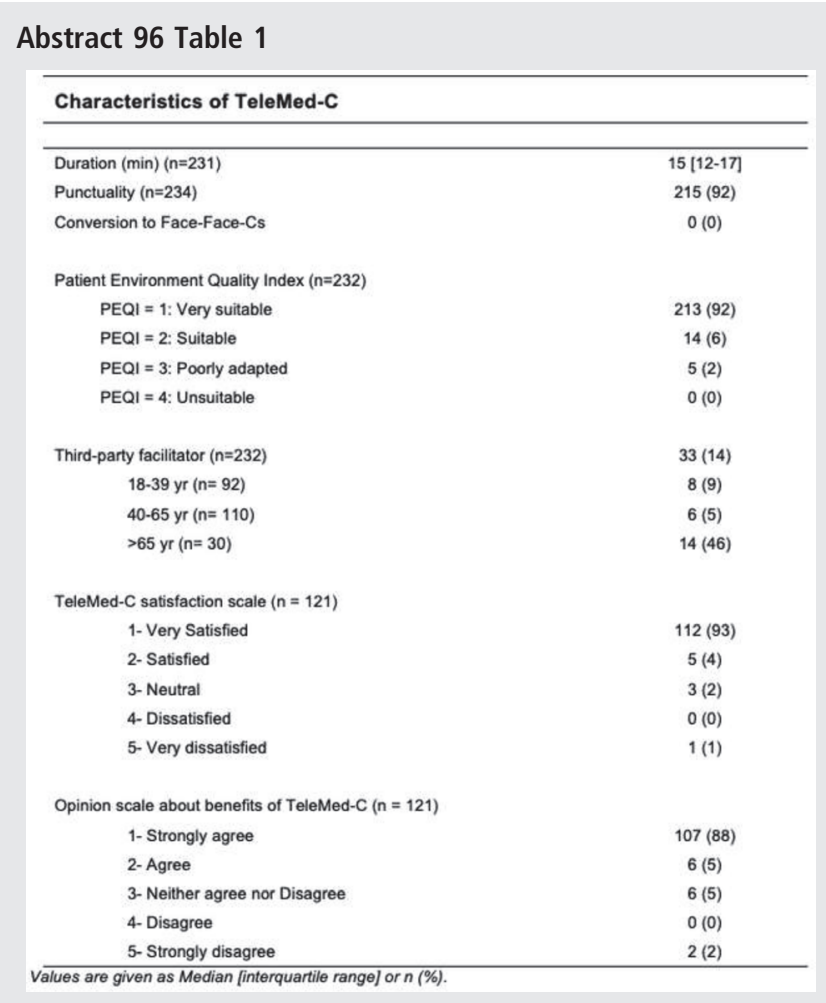

cancelled surgery. It should be considered as a valid procedure, and not only as an alternative to Face-Face-C.

\section{ABDOMINAL WALL BLOCKS FOR EMERGENCY ILEOSTOMY OPERATION IN A PATIENT WITH COVID-19 PNEUMONIA}

BB Güven, T Erturk, T Güner*, A Ersoy. Sultan Abdulhamidhan Sample and Training Hospital, Istanbul, Turkey

\subsection{6/rapm-2021-ESRA.97}

Background and Aims The anesthesia method to be administered during emergency surgical procedures for COVID-19 pneumonia patients carries great importance for both patient and surgical team. Regional blocks are generally used to ensure postoperative analgesia after abdominal surgery with general anesthesia. In this case, involving a patient receiving anticoagulant treatment due to COVID-19 pneumonia with planned emergency operation, the aim was to present the anesthesia management with rectus sheath(RS) and transversus

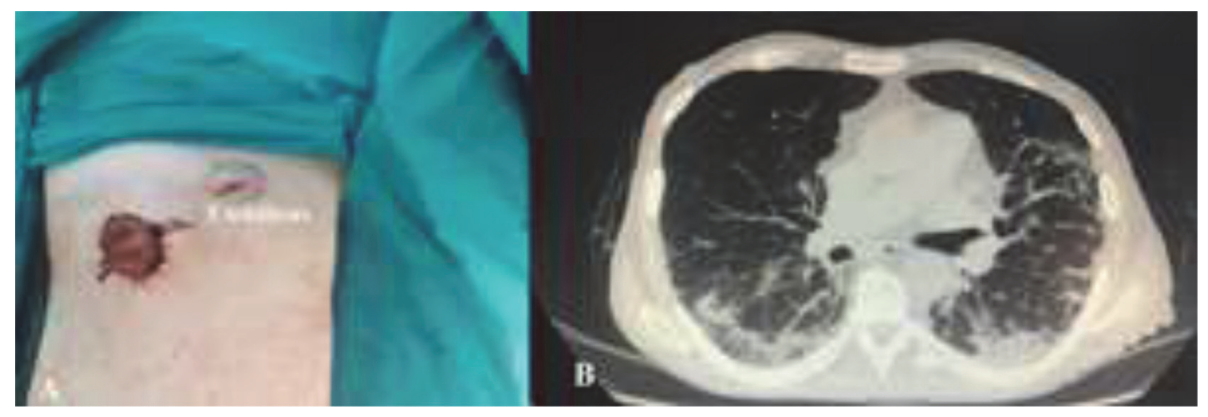

